\title{
Les Répercussions d'Un Réseau Ethnique Et La Dynamique Entrepreneuriale Des Libanais En Côte d'Ivoire
}

\author{
Ouattara Mohamed Zanga, (Doctorant) \\ Professeur Aloko-N'guessan Jérôme
}

Géographie Humaine et Economique / Institut de Géographie Tropicale (IGT), UFR des Sciences de l'Homme et de la Société, Université Félix

Houphouët-Boigny d'Abidjan, Côte d'Ivoire

Doi:10.19044/esj.2018.v14n16p180 URL:http://dx.doi.org/10.19044/esj.2018.v14n16p180

\begin{abstract}
Our objective in this article is to understand the characteristics of the entrepreneurship of the population of Lebanese origin in Côte d'Ivoire from the perspective of the ethnic network. In other words, it is about appreciating impacts of the Lebanese ethnic network on the development of their entrepreneurial activities, by scrutinizing the modalities such as the support for the immigration, the departure in business, the discovery of the entrepreneurial opportunity, the financing of the entrepreneurship and even the motive in the entrepreneurial commitment. To this end, this article joined within the framework of the studies relative to the ethnic and/or immigrant entrepreneurship.

This research thus left the Anglo-Saxon literature on the ethnic entrepreneurship and a field survey to seize how the ethnic network constitutes a major element of the economic integration of the Lebanese by the entrepreneurship in Côte d'Ivoire. In fact, their ethnic network of the Lebanese boosts their entrepreneurship through a shape of selection in the immigration; the family unit - by the tourism or the visit of the family - organizing the arrival of the new migrant until the obtaining of its first employment pending the acquisition of the Ivorian experience or the capital of the future business and the discovery of the entrepreneurial opportunity.
\end{abstract}

Keywords: Côte d'Ivoire, Entrepreneurial dynamics, Ethnic entrepreneurship, Lebanese, Ethnic network

\section{Résumé}

Notre objectif dans cet article est d'appréhender les caractéristiques de l'entrepreneuriat de la population d'origine libanaise en Côte d'Ivoire sous 
l'angle du réseau ethnique. En d'autres termes, il s'agit d'apprécier les retentissements du réseau ethnique libanais sur le développement de leurs activités entrepreneuriales, en scrutant les modalités telles que le soutien à l'immigration, le départ en affaire, la découverte de l'opportunité entrepreneuriale, le financement de l'entrepreneuriat et même le motif à l'engagement entrepreneurial. Pour ce faire, cet article s'est inscrit dans le cadre des études relatives à l'entrepreneuriat ethnique et/ou immigré.

Cette recherche est donc partie de la littérature anglo-saxonne sur l'entrepreneuriat ethnique et une enquête de terrain pour saisir comment le réseau ethnique constitue un élément majeur de l'intégration économique des Libanais par l'entrepreneuriat en Côte d'Ivoire. En fait, leur réseau ethnique des Libanais dynamise leur entrepreneuriat à travers une forme de sélection à l'immigration ; la cellule familiale - par le tourisme ou la visite de la famille - organisant l'arrivée du nouveau migrant jusqu'à l'obtention de son premier emploi en attendant l'acquisition de l'expérience ivoirienne ou du capital d'affaire future et la découverte de l'opportunité entrepreneuriale.

Mots-clés : Côte d'Ivoire, Dynamique entrepreneuriale, Entrepreneuriat ethnique, Libanais, Réseau ethnique

\section{Introduction}

En Afrique subsaharienne, la population d'origine libanaise est connue pour sa propension à s'engager dans les activités entrepreneuriales. En effet, elle est présente dans de nombreux secteurs d'activité, notamment en Côte d'Ivoire où elle représente la plus grande communauté d'Afrique. L'ampleur et la diversité de leur engagement entrepreneurial dans ce pays font que l'on parle aisément d'esprit d'entreprise des Libanais. L'esprit d'entreprise étant une notion complexe à définir et à cerner - «les économistes et les chefs d'entreprise ne s'accordant pas sur sa définition (...) tout en étant tous unanimes à considérer cette dynamique comme l'un des piliers de la croissance économique et de la création d'emplois dans toute société » (Holden, 2007) - nous adoptons celle de la dynamique entrepreneuriale. Cette dernière, au sein de communauté d'origine étrangère dans leur pays d'accueil, est ordinairement expliquée à partir de trois approches majeures (culturaliste, structuraliste et situationnelle) auxquelles l'on ne manque pas d'ajouter le fait capital de l'existence du réseau "ethnique"10. "L'accès [à celui-ci] en tant que source de financement, de main d'ouvre, d'approvisionnement, d'information et de conseils mais aussi en tant que marché explique d'une façon prépondérante le dynamisme entrepreneurial de certains groupes d'origine

${ }^{10} \mathrm{Il}$ est à noter que " "ethnique" est associé à un groupe (...) dont les membres ont une certaine conscience de leur appartenance à un groupe et à une commune origine et culture, ou (...) les autres les perçoivent comme ayant ces attributs » (Yinger, 1985). 
immigré » selon Brenner, Ramangalahy, Filion, Menzies et Amit (2000, p.21). De ce fait, la contribution du réseau ethnique à la dynamique entrepreneuriale au sein de communautés "ethnique" est perceptible par son apport d'une part au démarrage d'activités économiques et d'autre part au succès de ces dernières. Par ailleurs, les contributions de l'entrepreneuriat des communautés d'origine étrangère à l'extension des activités économiques de leur milieu d'accueil ne sont plus sous-estimées, désormais. «Parmi ces contributions, diverses études ont souligné la revitalisation du commerce (Lachman et Brett, 1996), le maintien de la vitalité des petites et moyenne entreprises (Waldinguer, 1989), la création d'entreprises par les femmes (Mesa et Boles, 1997), l'accélération des exportations (Haed et Ries, 1998) et la création d'emplois (Light et Rosenstein, 1995), etc. » (Filion, Brenner, Ramangalahy et Menzies, 2003, p.42). Dans le but donc d'apprécier les facteurs explicatifs du développement d'activités entrepreneuriales des Libanais en Côte d'Ivoire, nous mènerons notre investigation sous l'angle du réseau ethnique plutôt que selon les trois principales approches du dynamisme entrepreneurial de communautés d'origine étrangère dans leur pays d'accueil.

En effet, s'inscrivant dans une démarche exploratoire et descriptive plutôt qu'explicative et théorique, notre objectif, en présentant cet article, est d'exposer les processus en jeu dans la dynamique de création (ou reprise) d'activités économiques - depuis la décision de départ à l'immigration jusqu'à la concrétisation de l'intention entrepreneuriale - au sein de la communauté d'origine libanaise en Côte d'Ivoire, après avoir expliciter le phénomène de l'entrepreneuriat de population d'origine immigrée. En d'autres termes, en se proposant d'analyser la dynamique entrepreneuriale des Libanais en Côte d'Ivoire, notre ambition est d'appréhender les mécanismes sollicités ou mis en jeu dans le développement de leurs actions entrepreneuriales. "L'entrepreneuriat, [étant] (...) au premier chef la consécration de l'esprit d'entreprise, de la volonté d'entreprendre dans les affaires »(Julien et Marchesnay, 1996, p.11), derrière chaque activité économique existante - un entrepreneuriat - subsiste à l'évidence un esprit d'entreprise et une suite d'actions mises en jeu pour sa concrétisation, y compris la sollicitation du réseau ethnique.

\section{Méthodologie}

En se référant dans un premier temps à la littérature - souvent anglosaxonne - sur l'entrepreneuriat ethnique et/ou immigré pour étaler la revue de la littérature, cette recherche se base, dans un second temps, sur une enquête que nous avons effectuée (Novembre 2015 à Janvier 2016) auprès d'une cinquantaine de répondants d'origine libanaise dans six villes de la Côte d'Ivoire : Bouaké, Daloa, Gagnoa, Korhogo, San-Pédro et Soubré. Le choix de ces villes n'étant pas anodin (Figure 1), retenons que Korhogo représente 
la plus grande ville du nord, Bouaké la deuxième grande ville du pays (après Abidjan) située au centre, San-Pédro le deuxième port du pays, les autres (Daloa, Gagnoa et Soubré) sont les régions cacaoyères. Toutefois, la question de la représentativité de l'ensemble de la communauté libanaise ne semble pas être négligeable, à priori, pour notre échantillon. A posteriori, l'option qualitative de cette enquête semble, de notre point de vue, enjoindre l'adéquation de l'échantillon aux objectifs de la recherche plutôt qu'à une représentativité.

Figure 1 : La carte administrative de la Côte d'Ivoire et les villes d'enquête

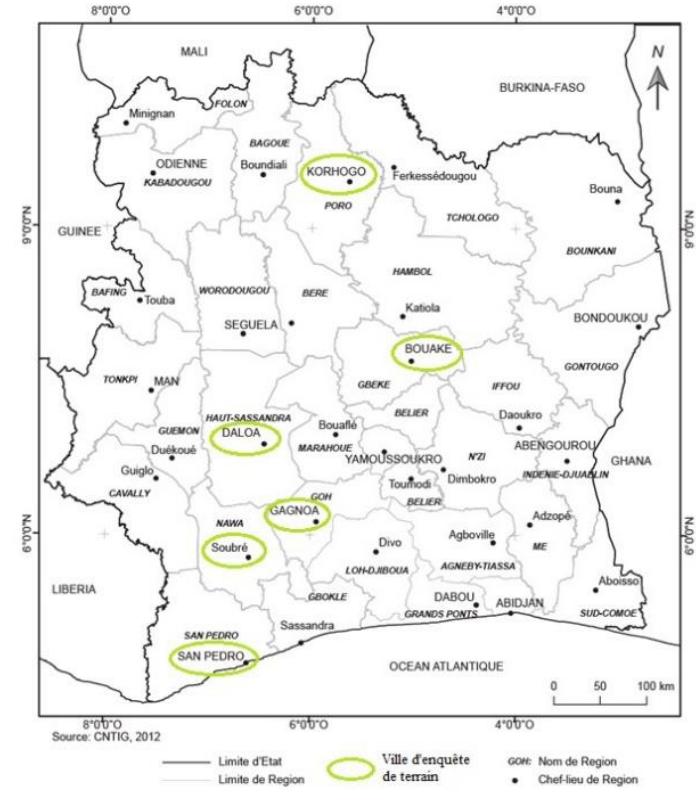

De ce fait, et confronté à une certaine méfiance de la population ciblée pour l'enquête ${ }^{11}$, la construction de notre échantillon s'est réalisée de façon raisonnée (empirique) et par convenance ; une méthode appropriée pour des études exploratoires comme celle-là. Pourtant, à cette méthode d'enquête, nous avons combiné une autre ; celle de boule de neige ${ }^{12}$ qui a consisté à se faire parfois aider (recommander) par un répondant pour trouver un individu (d'origine libanaise) pouvant être favorable à intégrer l'enquête en répondant aux questions.

${ }^{11}$ En dépit d'une demande formulée à l'endroit de la Chambre de Commerce et d'Industrie Libanaise de Côte d'Ivoire, il ne nous a pas été possible d'enquêter des entrepreneurs qui y sont inscrits. Il faut ajouter à cela, tous les autres refus individuels que nous avons essuyés.

${ }^{12}$ Cette méthode, lorsque qu'elle est utilisée seule, est associée à un risque de biais induit par une possible sélection des enquêtés pouvant être reliés à un sous-réseau limité comme étant du même secteur d'activité, etc. Cependant, elle présente l'avantage de pouvoir augmenter rapidement la taille de l'échantillon potentiel pour les besoins d'une recherche à l'intérieur d'une communauté ethnique. 


\section{Revue de la littérature}

A partir d'un ensemble de littérature sur l'entrepreneuriat ethnique et/ou immigré, quasiment anglo-saxonne, il s'agit dans cette section d'exposer les notions (approches, théories et modèles) nécessaires à l'appréhension du phénomène de l'acquisition d'unités économiques (des entreprises ou des activités économiques) - l'entrepreneuriat - au sein d'une communauté d'origine étrangère dans son pays d'accueil.

En effet, l'entrepreneuriat ethnique et/ou immigré semble mieux présenter ce phénomène de l'étude qui prend en compte le fait d'immigrer et surtout de s'intégrer économiquement par la mise en place d'activité économique dans le pays d'accueil. Et «si la majorité des recherches de ce champ portent sur les pays du Nord, le phénomène de l'entrepreneuriat ethnique [et/ou immigré] existe dans tous les pays qui reçoivent des flux d'immigration et où plusieurs groupes ethniques cohabitent » (Liègeois, 2007). Plutôt que l'entrepreneuriat immigré, notre choix se porte spécifiquement sur l'entrepreneuriat ethnique qui traduit le fait que " "ethnique" [étant] associé à un groupe (...) dont les membres ont une certaine conscience de leur appartenance à un groupe et à une commune origine et culture, ou (...) les autres les perçoivent comme ayant ces attributs » (Yinger, 1985, p.112) dans une société d'accueil donnée. En revanche, l'entrepreneuriat immigré renvoie à la notion de création d'activités économiques dans une société d'accueil UNIQUEMENT par un groupe (d'origine étrangère) issues DIRECTEMENT de l'immigration ; c'est-à-dire la première génération puisque les autres ne sont pas des immigrés au sens stricte du terme.

\section{Les approches de l'entrepreneuriat ethnique}

Pour tenter d'expliquer le phénomène de l'entrepreneuriat ethnique, différents auteurs tels que Ma Mung et Guillon (1986), Toulouse et Brenner (1988), Light et autres (1992), etc., l'ont présenté en l'attribuant aux ressources culturelles comme la religion ou certaines pratiques sociales du groupe « ethnique » des entrepreneurs (leur communauté). Par exemple, pour Toulouse et Brenner (1988) «un immigrant créera une entreprise parce que cette activité est hautement évaluée dans son groupe culturel »(p.6). D'autres auteurs considèrent plutôt que l'entrepreneuriat ethnique résulte de l'environnement socio-économique de leur pays d'accueil; parmi eux Bonacich (1973, 1977, 1980, 1987) ; Light (1979) ; Portes et Bach (1985) ; Portes (1987) ; Pyong-Gap (1984) ; Helly et Ledoyen (1994). De ce fait, deux principales approches ont guidé le corpus de l'entrepreneuriat ethnique dès ses débuts au cours de la décennie 1970 avec des auteurs tels que Light (1972, 1979), Bonacich $(1973,1977)$ : l'approche culturelle et l'approche structurosituationnelle (structurelle et "désavantage social"). Cette dernière approche 
a la particularité de renfermer deux tendances, à savoir une qui considère déterminantes les structures sociales, politiques et économiques du pays d'accueil, et l'autre les relations sociales entre le groupe «ethnique » et sa société d'accueil.

\section{Les théories de l'entrepreneuriat ethnique}

Il apparait dans la documentation anglo-saxonne sur l'entrepreneuriat ethnique que trois principales théories sont largement utilisées : la théorie des minorités intermédiaires (middelman minority theory), la théorie de l'enclave ou de la niche ethnique (ethnic enclave theory) et la théorie du désavantage ou de discrimination. Ces dernières mettent en relief les difficultés socioéconomiques que l'immigré rencontrerait dans sa société d'accueil; des difficultés le poussant à se replier sur sa communauté (le recours aux ressources ethniques) pour réaliser son projet. Ainsi, ces théories permettent de rendre compte de l'entrepreneuriat du groupe « ethnique » selon que celuici souhaite ou non s'intégrer (se sédentariser) dans son pays d'accueil. Par ailleurs, elles laissent apparaitre l'entrepreneuriat ethnique comme le fruit de l'existence de réseaux sociaux ethniques d'appui déclinés sous diverses formes relationnelles (familiale, personnelle, professionnelle, communautaire).

\section{Les modèles de l'entrepreneuriat ethnique}

A partir de théories, les chercheurs ont tenté de modéliser l'entrepreneuriat en contexte migratoire et deux modèles se sont révélés plus représentatifs du fait de leur adoption par plusieurs chercheurs : le modèle de Toulouse et Brenner $(1988,1990)$ et le modèle de Waldinger, Aldrich et Ward (1990). Notons que le second modèle est généralement appelé "modèle interactif de développement d'entreprises ethniques".

\section{* Le modèle de Toulouse et Brenner (1990)}

A partir de la convergence de trois théories explicatives (la théorie du désavantage, la théorie de la division du marché et la théorie culturelle), ce modèle suggère que certains groupes d'immigrés démontrent une propension plus grande que d'autres à créer des entreprises. Par ailleurs, le succès de ces immigrants repose sur les ressources du groupe ethnique d'une part et de leur tendance à se spécialiser dans des secteurs particuliers, notamment celui des services, d'autre part. Pour finir, Toulouse et Brenner (1990) observent que les motivations de l'entrepreneuriat immigré (création ou reprise d'entreprise) sont issues du désir de l'immigré d'être intégré dans la société d'accueil (désavantage), de la valorisation de l'activité entrepreneuriale par le groupe ethnique (culture) et de l'exploitation par l'immigrant des niches ou enclaves délaissées par la société d'accueil (division des marchés). 
* Le modèle de Waldinger et autres (1990)

Waldinger et autres proposent un modèle interactif - l'interaction entre les opportunités et les caractéristiques des groupes ethniques - dans lequel la stratégie d'entrepreneuriat ethnique est la résultante de quatre facteurs : les conditions du marché, les possibilités d'accès à la propriété, les prédispositions du groupe ethnique à entreprendre et la mobilisation des ressources. En effet, selon ces auteurs, «les structures d'opportunité sont déterminées par les caractéristiques du marché qui favorise ou non des produits et services destinés aux membres d'un groupe ethnique, mais aussi au marché non ethnique. Ces opportunités concernent aussi la capacité d'accès à l'entrepreneuriat, qui dépend de la compétition entre groupes ethniques, et des politiques publiques. Les caractéristiques des groupes concernent les éventuelles prédispositions à l'entrepreneuriat (culture, ambition, éducation, etc.), la capacité des groupes à mobiliser les ressources existantes, les réseaux sociaux ethniques, la capacité organisationnelle, ainsi que les politiques gouvernementales qui facilitent ou entravent la mobilisation des ressources 》 (Liégeois, 2007).

A l'instar des théories notées ci-avant, ces deux modèles laissent apparaitre la place non-négligeable du réseau ethnique - les ressources du groupe ethnique selon Toulouse et Brenner (1990) et les réseaux sociaux ethniques selon Waldinger et autres (1990) - dans l'entrepreneuriat ethnique.

\section{Le réseau ethnique dans l'entrepreneuriat ethnique}

Le réseau ethnique peut être défini comme un maillage des personnes de même origine «ethnique » qui sont reliés les uns aux autres sous diverses formes relationnelles (familiale, personnelle, professionnelle, communautaire). "Pour de nombreuses études en entreprenariat, le réseau de relations personnelles est une question centrale car, celui dans lequel est inséré l'entrepreneur immigré joue un rôle sur le fait de s'engager dans l'acte entrepreneurial et sur la réussite de cet acte »(Simen, 2015, p.181). En effet, en renchérissant Granovetter (2000), nous notons que les actions qu'ils [les entrepreneurs immigrés] entreprennent pour atteindre un objectif [entrepreneurial] sont encastrées dans des systèmes concrets, continus de relations sociales [ethniques] (p.84). Ce réseau ethnique, lorsqu'il existe ${ }^{13}$, constitue l'un des principaux facteurs explicatifs de l'entrepreneuriat de groupes ethniques (communauté d'origine immigrée) dans leurs sociétés

13 «Les communautés établies depuis longtemps présentent l'avantage de disposer de réseaux d'institutions et de liens sociaux plus structurés (Tenenbaum, 1986) contribuant à la mobilité de l'emploi, au renforcement des liens entre les acteurs mais également à faciliter l'ascension de membres de la communauté vers les sphères de la société d'accueil et l'accès aux ressources par les entrepreneurs qui seraient autrement impossibles à obtenir à l'intérieur du réseau formel d'accueil. » (Brenner et autres, 2000, p.3). 
d'accueil, comme le témoignent d'ailleurs Toulouse et Brenner (1988). Par ailleurs, en mentionnant la dynamique entrepreneuriale fondée sur l'activation du réseau social constitué essentiellement de «liens forts》 et de «liens faibles », Aldrich et Zimmer (1986) considèrent d'une part ceux-là - les liens forts composant le réseau ethnique - comme vecteur d'une forte propension à l'entrepreneuriat et facteur influençant la probabilité de succès, et d'autre part, ceux-ci comme instrument d'accès aux ressources et d'identification des opportunités. Enfin, "les recherches démontrent généralement que la création d'entreprises ethniques est centrée sur le réseau familial et intraethnique » (Brenner et autres, 2000, p.2) ; les «liens familiaux, régionaux, de dialecte et, finalement, ethniques [donnant] accès à un traitement de faveur » (Bonacich, 1973). Pour les auteurs de ces recherches, le financement nécessaire à la création d'activité, par tout immigré n'en disposant pas et ne recourant pas au service d'aide nationale de leur société d'accueil, est obtenu au sein de sa communauté (la famille, les relations ou les institutions) grâce à des prêts consentis. De ce fait, Pécoud (2004), en se référant à certains auteurs dont Aldrich et Waldinger (1990), mentionne que «l'appartenance à [un réseau ethnique] permet de surmonter les obstacles individuels à l'accès à l'entrepreneuriat» (p.14).

\section{Résultats et analyses}

Le réseau ethnique étant l'un des principaux facteurs explicatifs de l'entrepreneuriat ethnique - la jonction de deux phénomènes ; l'immigration et l'entrepreneuriat - nos résultats et analyses, scindés en deux grands points, présentent d'abord l'impact du réseau ethnique sur l'immigration des Libanais en Côte d'Ivoire, avant d'étaler ensuite son soutien à l'entrepreneuriat via l'emploi, le co-entrepreneuriat et la découverte de "l'opportunité entrepreneuriale $\rangle^{14}$. Toutefois, afin d'évaluer l'effet du réseau ethnique (notamment familial) sur les motivations à l'engagement entrepreneurial de ces Libanais, un dernier sous-point viendra questionner la création d'emploi pour soi-même ou pour d'éventuels membres de la famille d'une part et de l'héritage familial d'autre part.

Les retentissements du réseau libanais sur leur immigration et leur départ en affaire

* Le soutien à l'immigration de parents d'origine libanaise

Si autrefois les affrontements confessionnels ont entrainé l'émigration des Libanais et leur immigration en Afrique particulièrement, les raisons de leur migration en Côte d'Ivoire ont des causes diverses, en ce qui concerne les

${ }^{14}$ L'opportunité entrepreneuriale peut être défini comme une notion liée à la conception néoclassique de l'économie (Kirzner, 1997) qui suggère un traitement efficient des informations et connaissances asymétriques; les individus ne possédant pas les mêmes informations et connaissances essentielles à l'entrepreneuriat. 
répondants à notre enquête. Les résultats (Figure 2) sur les motifs de leur migration ne concernent évidemment que les répondants nés hors du pays (36 individus d'où $72 \%$ de notre échantillon). Notons qu'il a été difficile ici de démêler la cause de "recherche d'opportunite" à celle de "tourisme et famille" ; très souvent l'immigré rejoint un parent en Côte d'Ivoire parce que ce dernier à une opportunité d'affaire qui peut être intéressante pour l'emploi du nouveau arrivant.

Un répondant nous disait que du fait de son prétendu banditisme au Liban, il a été décidé par ses parents de l'envoyer chez son oncle en Côte d'Ivoire car celui-ci avait du travail pour lui (le neveu) afin qu'il sache que dans la vie, il faut travailler pour vivre. Le neveu (notre répondant) n'avait aucune activité au Liban.

Une autre répondante nous signifiait qu'elle est venue rendre visite à sa sœur qui était mariée à Abidjan. Grâce à sa sœur et son mari qui travaillaient dans une société libanaise, elle a eu une opportunité de travail qui a fait qu'elle est restée en Côte d'Ivoire.

Ainsi, le motif de la migration "tourisme et famille" semble être lié à un objectif de prospection personnelle de terrain. Le motif de "tourisme et famille" reste la caractéristique première de l'immigration libanaise en Côte d'Ivoire (47\%) et après vient la recherche d'opportunité d'affaire (31\%). Les raisons politiques au Liban, qui ont certainement motivées autrefois l'immigration d'un bon nombre arrivants dans le pays, tendent à s'amenuiser au fil des années pour laisser la place à une forte attraction liée à la force des liens ; notamment les "liens forts" de Granovetter (1973).

Figure 2: Répartition des enquêtés selon les motifs de leur migration

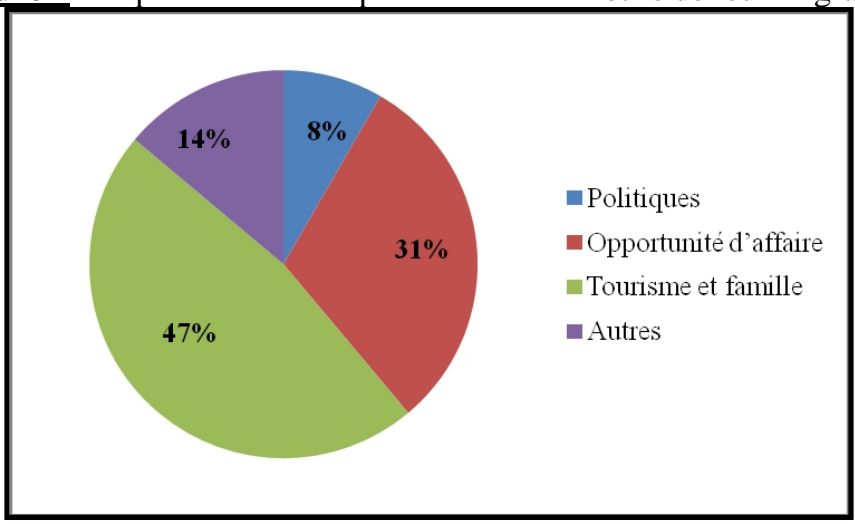

Source : Résultats de notre enquête de terrain

A travers les motifs de "tourisme et famille" et celui d'“opportunité d'affaire" des répondants, le réseau ethnique des Libanais se révèle finalement 
telle une filière à l'immigration ou de «chaîne migratoire $»^{15}$, puis telle une filière d'aide à l'installation de membre de la famille proche ou même éloignée.

Le support indispensable à l'entrepreneuriat libanais via l'emploi

Il s'agit ici de montrer en quoi le corollaire de l'immigration des Libanais à travers leur réseau ethnique est un soutien essentiel à leur entrepreneuriat via leur emploi par des membres de leur communauté lorsqu'ils ne sont pas co-entrepreneurs (associés). En d'autres termes, nos répondants sont-ils des employés, des associés ou les propriétaires des enseignes que nous avons visitées lors de notre enquête ? Les résultats obtenus (Tableau 1) tendent à vérifier les précédents ; ceux des motifs de migrations.

En effet, si les enseignes sont des propriétés libanaises, les trois cinquième $(3 / 5)$ de nos enquêtés y sont des employés alors que les deux cinquième $(2 / 5)$ restants de notre échantillon sont des associés et des propriétaires à hauteur de $10 \%$ et de $30 \%$ respectivement. Parmi ceux qui sont employés, certains le sont par leurs parents proches (le père, l'oncle, le frère et même le mari pour des femmes). Quant à ceux qui sont propriétaires, l'on trouve parmi eux des héritiers de propriétés familiales. Quelques-uns de ces héritiers sont des associés. D'ailleurs, les associés sont souvent liés par des liens forts tels que père et enfants, mari et femme, entre enfants, ou même entre amis de longtemps date.

Tableau 1: Répartition des enquêtés selon ce qui les lie aux enseignes

\begin{tabular}{|c|c|c|c|c|}
\hline & Propriétaires & Associés & Employés & Total \\
\hline Personnes enquêtées & 15 & 5 & 30 & 50 \\
\hline Proportions (\%) & 30 & 10 & 60 & 100 \\
\hline
\end{tabular}

Source : Résultats de notre enquête de terrain

A travers l'emploi et le co-entrepreneuriat (être des associés), l'entrepreneuriat libanais en Côte d'Ivoire répond à l'emploi d'une partie considérable de la population de leur communauté du pays bien qu'il permette par le jeu des externalités positives (directes ou indirectes) à l'emploi de diverses populations du pays. Par ailleurs, l'aspiration à se mettre à son propre compte dès que la bonne affaire se présentera, nous a été manifesté par près de trois quarts (3/4) des employés que nous avons rencontré. D'ailleurs, les parcours entrepreneuriaux des propriétaires et associés, nous enseignent qu'ils sont passés par l'emploi avant de se mettre à leur propre compte. Concernant les 20 propriétaires-associés, la moyenne du fait d'avoir changé d'emploi avant leur propre activité est de 1,5 fois, en notant qu'elle est séparément de 2 fois et 1,33 fois respectivement pour les associés et les propriétaires.

15 «C'est-à-dire que d'anciens migrants aident les nouveaux à venir, qui à leur tour aideront d'autres à venir et ainsi de suite. »(Weber, 2004, p.38). 


\section{Les déterminants de l'initiative entrepreneuriale des Libanais à travers leur réseau ethnique}

* La découverte de l'opportunité entrepreneuriale

A partir de l'approche de l'opportunité d'affaire développée par

Kirzner (2005 [1973]) - une autre vision de la théorie de l'innovation de Schumpeter - pour expliquer l'entrepreneuriat, des auteurs tels que Shane et Venkataraman (2000) suggèrent une perception de l'entrepreneuriat totalement attaché à l'identification, l'évaluation et l'exploitation d'opportunités d'affaires fructueuses. Par conséquent, l'on peut attester que « la découverte de l'opportunité [d'affaire] constitue une étape critique dans le processus entrepreneurial en ce sens qu'elle permet à l'entrepreneur potentiel de concrétiser en actions une idée d'affaires » (Filion et autres, 2003, p.51). Ces entrepreneurs potentiels, s'ils appartiennent à une communauté ethnique, utilisent en général trois types de ressources pour découvrir les opportunités: "leur capital humain individuel, leurs réseaux et leurs connaissances du secteur acquises soit par expérience, soit par études et analyses » (Filion, Brenner, Dionne et Menzies, 2007, p.49).

Dans cette recherche, afin d'évaluer la découverte de l'opportunité entrepreneuriale, nous allons utiliser les résultats d'enquête en ce qui concerne l'acquisition de compétences requises pour se lancer en affaire (une activité économique). Pour ce faire, rappelons que le niveau de scolarité des personnes d'origine libanaise en Côte d'Ivoire a beaucoup évolué depuis les premiers arrivés. Nos résultats montrent que les $90 \%$ des répondants ont au moins un niveau du secondaire. Toutefois, il s'avère que les engagements dans l'activité entrepreneuriale ne se font quasiment pas dans le domaine de formations acquises. Les propriétaires et associés que nous avons rencontré, ont tous été, d'une façon ou d'une autre, employés par quelqu'un et parfois dans divers secteurs d'activité avant de se lancer dans leurs propres affaires. Etre employé avant son initiative entrepreneuriale répond vraisemblablement à la recherche d'acquisition une certaine compétence et du capital indispensables à son entrepreneuriat, mais surtout cela laisse le temps de se faire connaitre davantage au sein du réseau ethnique existant en attendant l'opportunité entrepreneuriale.

Par ailleurs, le dépouillement du questionnaire, relatif aux réponses à la question ouverte sur "comment avez-vous découvert cette opportunité d'affaire?", nous renseigne que les découvertes se sont faites de diverses manières et souvent par l'association de différents facteurs. Pourtant, il ressort que le réseau d'information, notamment celui constitué par les liens forts au sein du réseau ethnique (contacts ethniques y compris la famille), est généralement la ressource la plus annoncée par les enquêtés.

Finalement, si nos répondants ont eu recours à leurs expériences professionnelles (les différents emplois effectués) pour acquérir des 
compétences, c'est plutôt le réseau ethnique qui aura "le dernier mot" quant aux découvertes de l'opportunité d'affaire. En d'autres termes, ils ont trouvé en majorité leurs occasions d'affaire en s'appuyant sur leurs connaissances du domaine d'activité entrepreneuriale en question; des connaissances liées à leurs expériences de travail et surtout au réseau ethnique d'information ${ }^{16}$. Audelà donc des caractéristiques des enquêtés (les entrepreneurs ethniques), le réseau ethnique à travers l'hétérogénéité des liens relationnels est déterminant à la découverte de l'opportunité entrepreneuriale.

* Le financement de l'entrepreneuriat

Le capital est l'élément clé de tout entrepreneuriat et sa source peut être diversifiée d'un entrepreneur à l'autre en fonction du montant nécessaire à l'investissement initial. Plutôt que le montant de l'investissement initial, nous avons cherché à savoir au cours de notre enquête, si le capital provenait de soi-même (épargne personnelle), des membres de la famille (héritage familial ou association avec un parent), des banques ou même de différentes autres combinaisons (autres).

$\underline{\text { Figure } 3}$ : Répartition des propriétaires et associés selon leur source de financement

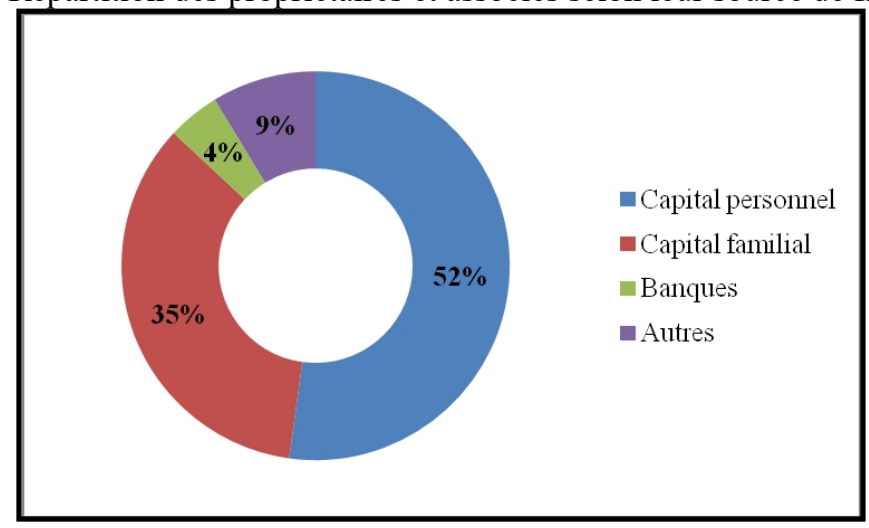

Source : Résultats de notre enquête de terrain

Il apparait (Figure 3) que le capital personnel est au-dessus de la moyenne $(52 \%)$ dans le financement de l'entrepreneuriat libanais. Il est suivi par le capital familial qui finance un peu plus d'un libanais sur trois (35\%) dans son entrepreneuriat. Les banques semblent être le dernier recours au financement de cet entrepreneuriat ethnique. En réalité, les banques sont généralement réticentes à prêter à une population d'origine immigrée sans l'assurance d'un garant (un aval financier) ; une démarche que l'entrepreneur ethnique contourne très souvent en s'associant à son éventuel garant ou en lui

${ }^{16}$ Des auteurs tels que Burt R.S. (1995), Granovetter M. (1995), Aldrich et Zimmer (1986), Chabaud D. et Ngijol J. (2005) (etc.) ont mené des réflexions sur la contribution du réseau social dans le processus d'identification d'opportunité en mentionnant l'importance des liens faibles. 
empruntant directement ou même à un autre (notamment les élites communautaires) par le jeu du réseau ethnique. Cette pratique est d'autant plus fréquente que le réseau ethnique est bien structuré.

* Les motivations des engagements entrepreneuriaux

Pour se lancer dans une activité économique, tout entrepreneur immigré doit développer des stratégies - les «prédispositions » de Waldinger et autres (1990) - qui prennent en compte le contexte économique local d'insertion d'une part et de son capital économique ou culturel individuel d'autre part. Se référant toujours à Waldinger et autres (1990), ce sont ces stratégies qui permettent à l'entrepreneur immigré de surmonter d'une certaine manière les problèmes fondamentaux à l'entrepreneuriat.

Dans le but d'appréhender d'éventuels effets du réseau ethnique à travers des modalités telles que "création d'emploi pour soi-même ou pour autres membres de sa communauté" et "héritage familial", il a été question d'ordonner quatre motivations prédéterminées de la plus importante (1) à la moins importante (4). Ces motivations à ordonner par chaque enquêté sont les suivantes : être son propre patron, l'auto-emploi ou la création d'emploi, la suite d'affaire familiale (héritage) et autres raisons. Par conséquent, une moyenne plus faible obtenue par une motivation signifie qu'elle est plus importante par rapport à une autre qui a obtenu une plus grande moyenne. Partant, il résulte des moyennes obtenues (Tableau 2) par chacune des raisons prédéterminées que le départ en affaire des répondants est motivé successivement par ordre de priorité par le fait d'abord d'être son propre patron $(1,85)$, ensuite par l'héritage familial $(2,10)$, après par autres motivations $(2,95)$ et enfin par la création d'emploi $(3,35)$. Il convient donc d'affirmer que la recherche d'autonomie (être son propre patron) et l'héritage familial sont les motivations majeures à l'entrepreneuriat libanais en Côte d'Ivoire.

Tableau 2 : Classement des motifs de départ en affaire des enquêtés

\begin{tabular}{|c|c|c|c|c|}
\hline Différentes raisons & $\begin{array}{c}\text { Etre son propre } \\
\text { patron }\end{array}$ & $\begin{array}{c}\text { Création } \\
\text { d'emploi (a) }^{\text {(a) }}\end{array}$ & $\begin{array}{l}\text { Héritage } \\
\text { familial }^{(b)}\end{array}$ & Autres \\
\hline Sommes totales & 37 & 67 & 42 & 59 \\
\hline Moyennes & 1,85 & 3,35 & 2,10 & 2,95 \\
\hline
\end{tabular}

(a) Création d'emploi pour soi ou pour d'autres et (b) Héritage familial à sauvegarder ou à laisser à ses progénitures.

Source : Résultats de notre enquête de terrain

Néanmoins, en ne comptabilisant que les premiers choix faits par les répondants et en prenant en compte les employés qui gèrent l'activité d'un 
parent proche ${ }^{17}$, il s'avère que la création d'emploi prend une importante proportion dans la motivation des répondants au départ en affaire. De ce fait, le rang précédent des motivations au départ en affaire est modifié (Figure 4) comme le suivant : d'abord être son propre patron (36\%), ensuite la création d'emploi (28\%), après l'héritage familial (24\%) et enfin autres motivations (12\%). Dans ce contexte donc, la recherche d'autonomie (être son propre patron) et la création d'emploi notamment pour les parents propres (les liens forts) sont les principales motivations à cet entrepreneuriat libanais du pays.

Figure 4 : Répartition des enquêtés selon les motifs de leur départ en affaire

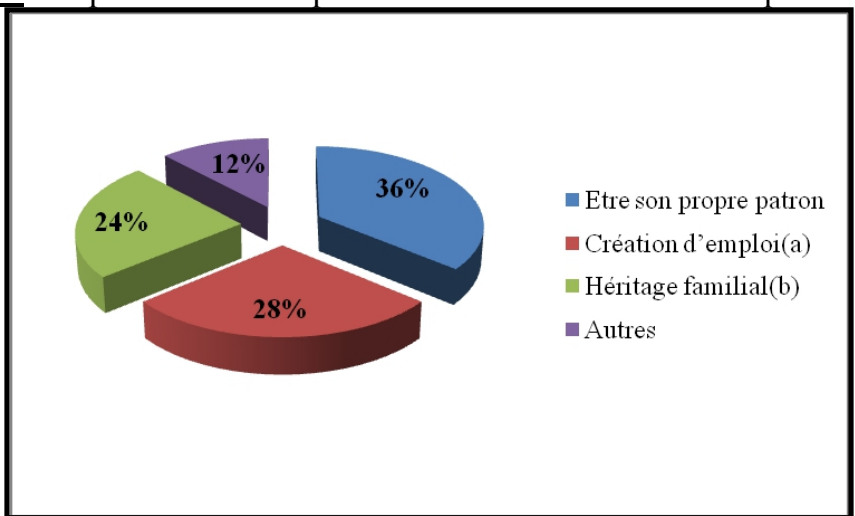

Source : Résultats de notre enquête de terrain

Il apparait donc une forme d'influence du réseau ethnique sur les motivations des engagements entrepreneuriaux des Libanais en Côte d'Ivoire. En effet, l'immigré arrive grâce à un réseau familial et trouve un emploi grâce à ce dernier qui s'étend au réseau ethnique, et cela, afin d'avoir les bases suffisantes (de l'expérience) et/ou les financements nécessaires à son entrepreneuriat.

\section{Synthèse et limites des résultats}

\section{La synthèse}

A l'observation des différentes théories explicatives de l'entrepreneuriat ethnique, il apparait un manifeste manquement dans l'explication de l'entrepreneuriat libanais en Côte d'Ivoire, en se référant qu'à une seule théorie. Toutefois, cette étude s'évertue à faciliter son appréhension sous l'angle du réseau ethnique.

En effet, le phénomène de l'entrepreneuriat en contexte migratoire (l'entrepreneuriat ethnique), à travers nos enquêtés, semble mieux se

${ }^{17}$ Dans ce cas, nous faisons l'hypothèse que ce départ en affaire n'aurait pas eu lieu si ce dernier (frère gestionnaire) n'était pas disponible ; c'est une création d'emploi déjà pour ce gestionnaire. 
comprendre à travers l'inspection attentive du réseau ethnique; chaque variable étudiée étant un module de son illustration.

En tenant compte des différents motifs d'immigration des enquêtés, ceux-ci révèlent que le réseau ethnique des Libanais est non seulement une filière à l'immigration de ses membres, mais aussi une filière d'aide à l'emploi ou à l'initiative entrepreneuriale de membres de la famille proche ou même éloignée. Le réseau ethnique libanais est de ce fait un support fondamental favorisant l'entrepreneuriat des Libanais, à travers les partenariats (associés) à l'engagement entrepreneurial, mais aussi à travers surtout 1'emploi des membres (proches ou éloignés) de la communauté. D'ailleurs, ces emplois des membres de la communauté libanaise leur confèrent des compétences pour se lancer à leurs tours dans la création d'activités économiques, dès que l'opportunité d'affaire se présentera, très souvent par les canaux de relations hétérogènes (familial, professionnel, institutionnel, etc.) du réseau ethnique d'information. En outre, ces emplois constituent la source des capitaux personnels nécessaires à toute création d'entreprise (unité économique) et concernant la communauté libanaise en Côte d'Ivoire, ces capitaux personnels sont largement suivis par les financements familiaux (héritage familial, partenariat avec un parent ou prêt auprès d'un parent). Par ailleurs, en scrutant les motivations à l'engagement entrepreneurial des enquêtés, "la création d'emploi pour soi ou pour d'autre" (surtout) et "l'héritage familial à sauvegarder ou à laisser à ses progénitures" qui sont telle une forme de création ou sauvegarde de réseau familial d'emploi ou d'entrepreneuriat, représente plus de la moitié (52\%) des motifs de leur départ en affaire.

\section{Les limites}

Comme dans quasiment tout travail de recherche, les résultats de cet article présentent des limites qui vont de la technique d'échantillonnage (la représentativité) à la méthode d'analyse. En effet, notre échantillon a été affecté par le fait que notre population d'étude soit difficile d'accès - notre demande auprès de la CCILCI ${ }^{18}$ restée sans suite - et discrète - une méfiance affichée à notre endroit ${ }^{19}$ - quand il s'est agi de nous renseigner sur leurs processus de création d'activités économiques. De ce fait, nous ne pouvons prétendre avoir obtenu des résultats généralisables à toute la population d'origine libanaise du pays, quoiqu'ils permettent d'en appréhender la dynamique entrepreneuriale d'un certain ensemble non-négligeable, sous l'angle du réseau ethnique : la population commerçante.

${ }^{18}$ La Chambre de Commerce et d'Industrie Libanaise de Côte d'Ivoire

${ }^{19}$ Nous avons été souvent pris pour d'un agent déguisé des impôts malgré la possession d'un document d'attestation de recherche doctorale, en plus de la promesse de l'anonymat et la confidentialité dans le traitement des données. Par ailleurs, nous avons dû abandonner certains questionnaires répondus à cause de l'évidence de réponses erronées. 
Quant aux éventuelles limites concernant les méthodes d'analyse, elles sont les corollaires du nombre limité de nos répondants (l'échantillon) qui a rendu difficile la réalisation de statistiques avancées sur de possibles relations ou liaisons existantes entre deux ou plusieurs variables. En effet, grâce aux pourcentages de l'écart maximum par exemple, il aurait été possible d'aller plus loin dans les statistiques pour observer s'il existe des relations positives entre les différentes variables ou modalités choisies.

Par ailleurs, une autre limite à cette enquête est d'avoir manqué de nombre suffisant d'enquêtés de sexe féminin afin d'en apprendre sur la manifestation de l'entrepreneuriat des femmes d'origine libanaise du pays, à travers le réseau ethnique. Les contraintes culturelles et notamment religieuses font qu'il est laborieux de rentrer en contact avec elles pour répondre aux questionnaires. Toutefois, il est certain que l'implication de la femme dans l'entrepreneuriat des personnes d'origine libanaise prend de plus en plus de l'ampleur et "bouleverse" les rapports existants entre elles et les hommes de leur communauté comme dans toutes communautés où il y a une "domination" masculine.

$\mathrm{Du}$ fait toujours de la méfiance de la population enquêtée, notre échantillon s'est limité finalement aux commerces ; un manque de diversité de secteur d'activité, même si le commerce représente plus de $70 \%$ des activités économiques entrepreneuriales des personnes d'origine libanaise en Côte d'Ivoire. Ainsi, nous réitérons que cela nous amène à n'avoir aucunement la prétention de généraliser les résultats de cet article à toute la communauté d'origine libanaise du pays.

\section{Conclusion}

A travers le réseau ethnique, l'entrepreneuriat constitue l'élément majeur de l'intégration économique de populations d'origine libanaise en Côte d'Ivoire. En effet, des données recueillies lors de notre enquête de terrain sur les activités économiques de cette population et leurs processus de création, les observations ont permis de comprendre que leur dynamique entrepreneuriale se structure depuis la cellule famille (tourisme et famille) qui organise l'arrivée du nouveau migrant jusqu'à son premier emploi (l'acquisition de l'expérience ivoirienne et/ou le capital d'affaire future) auprès d'un membre de la communauté libanaise grâce à leurs activités entrepreneuriales. D'ailleurs, l'apport personnel dans la mise en place de son unité économique (entreprise) est généralement suivi par l'apport familial.

Par ailleurs, d'autres observations offrent la possibilité d'apprécier qu'en dépit du fait qu'être son propre patron suscite généralement l'engagement entrepreneurial des populations libanaises du pays, ces dernières participent largement au maintien de leur réseau ethnique par leur volonté de créer des emplois ou activités pour les membres proches ou éloignés de leurs 
familles d'une part et de sauvegarder ou de laisser un héritage familial à leurs progénitures d'autre part.

\section{References:}

1. Aldrich H.E. et Waldinger, R.D. (1990), Ethnicity and entrepreneurship, In: Annual Review of Sociology, vol.16, $\mathrm{n}^{\circ} 1, \mathrm{pp} .111-$ 135.

2. Aldrich H.E. et Zimmer C. (1986), Entrepreneurship through social networks, In: The Art and Science of Entrepreneurship, Ballinger, Donald Sexton and Raymond Smilor, pp.3-23.

3. Bonacich E. (1973), A Theory of Middleman Minorities, In: American Sociological Reviews, vol.38, n5, pp.583-594 // Bonacich E. (1977), The Past, Present and Future of Split Labour Market Theory, In: Research in Race and Ethnic Relations, vol.1, pp.17-64 // Bonacich E. et Jung T.H. (1982), A portrait of Korean small business in Los Angeles: 1977, In: Koreans in Los Angeles: Prospects and Promises, pp.75-98 // Bonacich E. (1980), Class Approaches to Ethnicity and Race, In: Insurgent Sociologist, vol.10, n², pp.9-23 // Bonacich E. (1987), Making It in America: A Social Evaluation of the Ethics of Immigrant Entrepreneurship, In: Sociological Perspectives, vol.30, $\mathrm{n}^{\circ} 4$, pp.446-466.

4. Brenner G.A., Ramangalahy C., Filion L.J., Menzies T.V. et Amit R.H. (2000), Entrepreneuriat ethnique et rôle des réseaux de soutien : les entrepreneurs chinois dans trois grandes villes canadiennes, In : Actes - Entrepreneurship, vol.21, n²1, pp.21-31.

5. Burt R.S. (1995), Le capital social, les trous structuraux et l'entrepreneur, In : Revue française de sociologie, vol.36, n4, pp.599628.

6. Chabaud D. et Ngijol J. (2005), La contribution de la théorie des réseaux sociaux à la reconnaissance des opportunités de marché, In : Revue Internationale P.M.E, vol.19, n¹, pp.29-46.

7. Filion L.J., Brenner G.A., Dionne L. et Menzies T.V. (2007), L'identification d'une occasion d'affaire en Contexte d'entrepreneuriat ethnique, In : Revue internationale P.M.E., vol.20, $\mathrm{n}^{\circ} 2$, pp.33-57.

8. Filion L.J., Brenner G.A., Ramangalahy C. et Menzies T.V. (2003), Démarrage d'entreprises par les Chinois, Italiens et Sikhs au Canada : quelques résultats empiriques, In : Cahier de recherche n²003-15 [Journal of Small Business and Entrepreneurship, vol.16, n³-4, pp.41-65].

9. Granovetter M. (Ed) (2000), Le marché autrement : les réseaux dans l'économie, Paris, Desclé de Brouwer, 239p. 
10. Granovetter M. (1973), The strength of weak ties, In: American Journal of Sociology, vol.78, $\mathrm{n}^{\circ} 6$, pp.1360-1380.

11. Granovetter M. (1995), The economic sociology of firms and entrepreneurs, In: Portes A. (Ed) The Economic Sociology of Immigration. Essays on networks, Ethnicity and Entrepreneurship, New York : Russel Sage Foundation, pp.128-165.

12. Helly D. et Ledoyen A. (Eds) (1994), Immigrés et création d'entreprise : Montréal 1990, Québec, IQRC, 305p.

13. Holden J. (Ed) (2007), Principles of Entrepreneurship, Washington: U.S. Department of State, Bureau of International Information Programs, 156p, [En ligne] http://onlinebooks.library.upenn.edu/webbin/book/lookupid?key=olb p54235

14. Julien P-A. et Marchesnay M. (Eds) (1996), L'entrepreneuriat, Paris, Economica, 112p.

15. Kirzner I. (Ed) (2005[1973]), Concurrence et esprit d'entreprise, Paris, Economica, 209p.

16. Liégeois L. (2007), L’entrepreneuriat ethnique du réseau Ville Région Monde, In : Réseau interuniversitaire d'études urbaines et spatiales, [En ligne] www.vrm.ca/Cap_0709.asp, Cité en Juillet 2007.

17. Light I. (Ed) (1972), Ethnic Enterprise in America: Business and Welfare among Chinese, Japanese and Blacks, Berkeley, University of California Press, 209p.

18. Light I. (1979), Disadvantaged Minorities in Self-Employment, In: International Journal Comparative Sociology, vol.20, $\mathrm{n}^{\circ} 1-2$, pp.31-45.

19. Light I., Sabagh G., Bozorgmehr M., Der-Martirosian C., BodyGendrot S., Ma Mung E. et Hodeir C. (1992), Los Angeles : l'économie ethnique iranienne, In : Revue européenne des migrations internationales, vol.8, $\mathrm{n}^{\circ} 1, \mathrm{pp} .155-169$.

20. Ma Mung E. et Guillon M. (1986), Les commerçants étrangers dans l'agglomération parisienne, In : Revue Européenne des Migrations Internationales, vol.2, $\mathrm{n}^{\circ} 3$, pp.105-134.

21. Pécoud A. (2004), Réseaux, ethnicité et institutions dans les économies immigrées, In : Revue Homes et migration, $\mathrm{n}^{\circ} 1250$, pp.13-23.

22. Portes A. et Bach R.L. (Eds) (1985), Latin Journey: Cuban and Mexican Immigrants in the United States, Berkeley, University of California Press, 432p.

23. Portes A. (1987), The Social Origins of the Cuban Enclave Economy of Miami, In: Sociological Perspectives, vol.30, n4, pp.340-372.

24. Pyong-Gap M. (1984), A Structure Analysis of Korean Business in the United States, in: Ethnic Group, vol.6, pp.1-25. 
25. Shane S. et Venkataraman S. (2000), The Promise of Entrepreneurship as a Field of Research, In: Academy of Management Review, vol.25, $\mathrm{n}^{\circ} 1, \mathrm{pp} .217-226$.

26. Simen S.F. (2015), Entreprenariat immigré et réseautage : cas des commerçants chinois installés au Sénégal, In : Recherches en Sciences de Gestion, n¹08, pp.179-210.

27. Tenenbaum S. (1986), Immigrants and capital: Jewish Loan Societies in the United States, 1880-1945, In: American Jewish History, vol.76, $\mathrm{n}^{\circ} 1, \mathrm{pp} .67-77$.

28. Toulouse J-M. et Brenner G.A. (1988), Les entrepreneurs immigrants : à la recherche d'un modèle théorique, In : Rapport de recherche, $\mathrm{n}^{\circ} 88$ 05-01, Montréal, EHEC, 32p.

29. Waldinger R.D., Aldrich H. and Ward R. (Eds) (1990), Ethnic entrepreneurs: immigrant business in industrial societies, London/New Delhi, Sage Publications, vol.1, 226p.

30. Weber S., 2004, « De la chaîne migratoire à la migration individuelle des Roumains à Rome ", In : Hommes et migration, Réseaux sociaux en migration, $\mathrm{n}^{\circ} 1250$, pp.38-48.

31. Yinger J.M. (1985), Ethnicity, In: Annual Review of Sociology, vol.11, pp.151-180. 